\title{
UV-VIS spectroscopic studies of some skin of tomatoes and peppers
}

\author{
Cheyma NACEUR ABOULOULA*, Amane OUERIAGLI, Abdelkader OUTZOURHIT \\ Nanomaterials for Energy and Environment Laboratory (N2EL), Facultyof Sciences, Semlalia, \\ P.O Box. 2390, 40000 Marrakesh, Morocco \\ *Corresponding author: E-mail: cheyma.naceur@ced.uca.ac.ma
}

Keywords: UV-Visible Spectroscopy, Skin, Tomatoes, Pepper, Bio-Polymers

\begin{abstract}
Vegetable skins have many properties that can be used to produce new materials. In this study, our work looks to exploit the bio-resources in the industry. We study the optical property of fresh and dried skin of tomato and pepper.
\end{abstract}

\section{Introduction}

Today, scientists are committed to increase the respect of the environment and exhaustible fossil resources. For that, an interesting alternative is the valorization our waste for the development of new bio-degradable materials for industry.

Skin of most of fruits and vegetables finished as waste in agro-industrial. These vegetable raw materials are rich in polymers having particularly interesting properties for the plastic industry, such as biodegradability, biocompatibility, and some editable physico-mechanical properties $[1,2,3]$.

Our work focuses on the study of some physico-chemical properties of some fruits to use it in construction of new materials. In this context, it was led to make a first characterization of tomatoes skin, peppers skin: red, yellow and green. This fruit consumed highly in our daily lives and which have a tangible reject rate usable in the industry for the manufacture of product with altered characteristics.

\section{Materials}

Tomatoes and pepper were obtained from the local market and maintained at room temperature $\left(25^{\circ} \mathrm{C}\right)$ until their use. Subsequently, the outer skin was separated from the rest of the sample with a blade, and then was placed on a glass substrate in a uniform manner. The samples are then used without any additional treatment. The optical transmittance measurements were performed using a Shimadzu UV-PCspectrophotometer in the 200-2800nm range. It was used to study the optical properties of our samples.

\section{Results and discussion}

At first, we use the UV spectrometry to define optical properties of the samples chose (Figure 1). We find that our samples absorb in the same frequency.

After that, we follow the behavior of our skins by takingthe transmission spectrum of the simple during 3 weeks: In the first week, spectrums are taken every day (D1, D2, D3, D4, D5, D6). In the second, they are taken every other day (D8, D10, D12). At the last week, we take a spectrum at the end of the week (D19, D26) (Figure 2). 


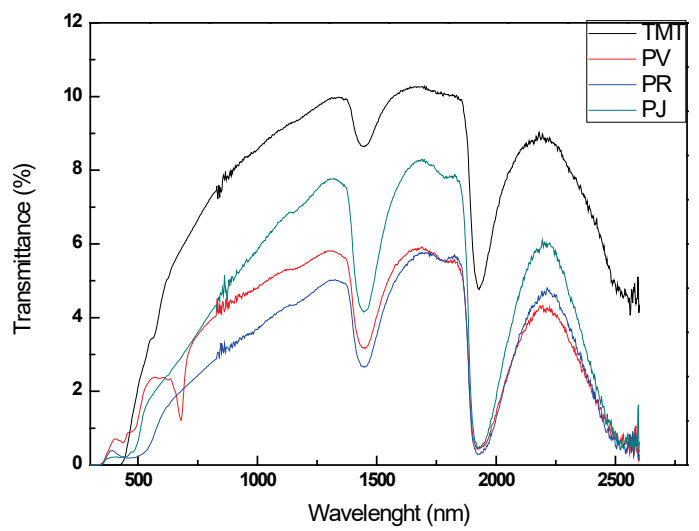

Fig. 1. Transmission spectrum of fresh skins (TMT: tomato, PV: green pepper, PR: red pepper and PJ: yellow pepper)

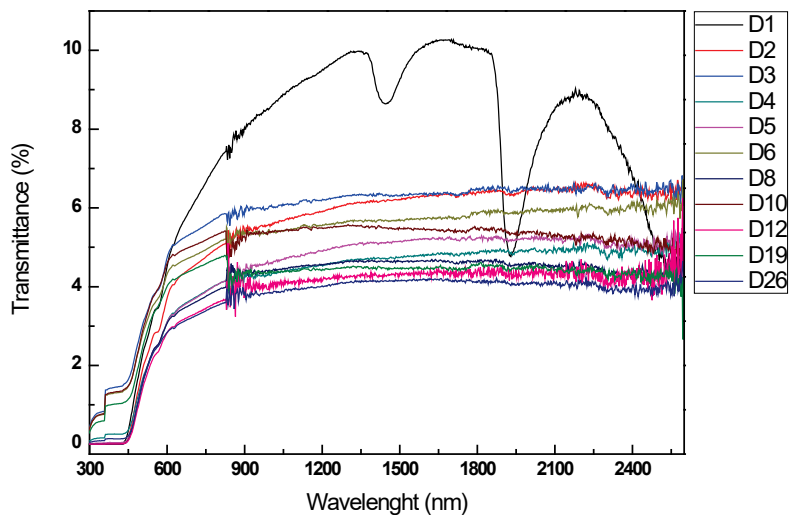

Fig.2. Evolution of transmission of tomato skin function of time

We note that the two peaks disappear at the $2^{\text {nd }}$ day (D2), the disappearance of these peaks, after dryly membranes, is attributed to the water molecule. The same remark is noted for all species studied. Using liquid water spectrum, it absorbs into two frequencies bands close to those of the skins studied with a shift due to the chemical groups associated with it (Figure 3).

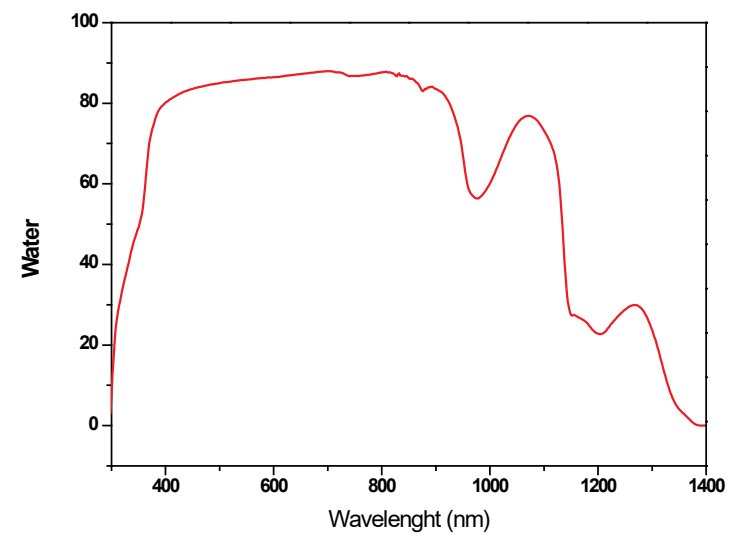

Fig.3. Transmission spectrum of liquid water 
To verify this hypothesis, we emerge dry skins in boiling water and taken up spectrum again (Figure 4). Comparing results of dry skin tip in water and fresh skin: we found that the peaks messing in drying state appear again; it shows that the peaks are related to the water molecule.
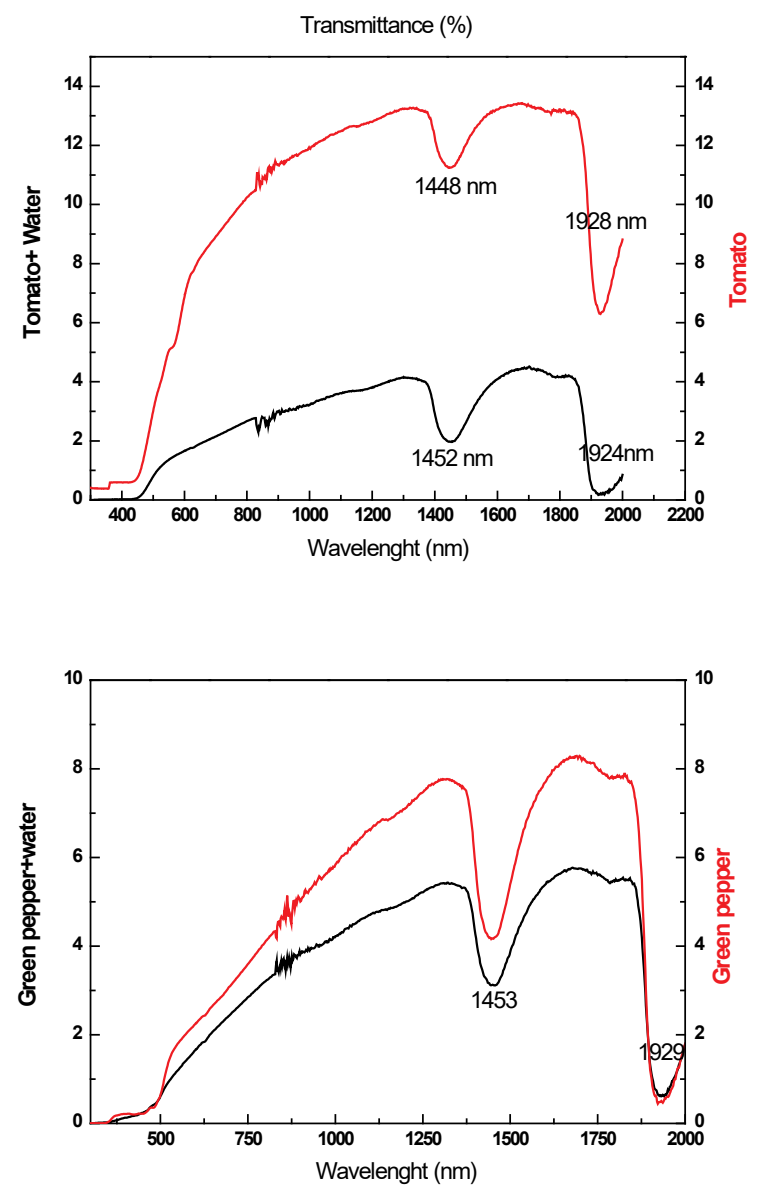

Fig.4. Absorption spectrum of dry skin immersed in boiling water

We can say that our skins absorb in the same wavelength of liquid water absorption.

\section{Conclusion}

As we have mentioned early, the studied skins have many useful properties in new manufacturing materials[4]. Studied skins absorb in the same absorption bands of the molecule of water, as they have the same chemical groups in infrared study.

Looking ahead, we will study mechanical and electrical/ dielectrical properties and the chemically composition of these membranes for their use in construction of new materials.

\section{References}

[1] Thielen, M.: Industrial Composting, "bioplastics MAGAZINE”, Vol. 4., Issue 02/2009,

[2] Gandini, Alessandro, "polymers from renewable resources, polymer chemistry", 2012,

[3] Belgacem, Monomers, "Polymers and Composites from Renewable Resources", 2008,

[4] http://triblive.com/business/headlines/6259292-74/heinz-based-ford 